\title{
AaTAS1 and AaMFS1 Genes for Biosynthesis or Efflux Transport of Tenuazonic Acid and Pathogenicity of Alternaria alternata
}

\author{
Fan Sun, ${ }^{1}$ Xueqiang Cao, ${ }^{1}$ Dianzhen $\mathrm{Yu},{ }^{1}$ Dongqiang Hu, ${ }^{1}$ Zheng Yan, ${ }^{1}$ Yingying Fan, ${ }^{2}$ Cheng Wang, ${ }^{2}$ \\ and Aibo $\mathrm{Wu}^{1, \dagger}$ \\ ${ }^{1}$ SIBS-UGENT-SJTU Joint Laboratory of Mycotoxin Research, CAS Key Laboratory of Nutrition, Metabolism and Food \\ Safety, Shanghai Institute of Nutrition and Health, University of Chinese Academy of Sciences, Chinese Academy of \\ Sciences, Shanghai, China \\ ${ }^{2}$ Institute of Quality Standards \& Testing Technology for Agro-Products, Xinjiang Academy of Agricultural Sciences, Key Lab \\ oratory of Agro-Products Quality and Safety of Xinjiang, Laboratory of Quality and Safety Risk Assessment for Agro-Products \\ (Urumqi), Ministry of Agriculture and Rural Affairs, Urumqi, China
}

Accepted 15 February 2022.

Taking tenuazonic acid (TeA) synthetase 1 (TAS1) in Pyricularia oryzae as a reference, the homolog AaTAS1 was first anchored in Alternaria alternata via de novo sequencing. Subsequently, AaMFS1, as a major facilitator superfamily (MFS) protein-encoding gene in the adjacent upstream region, was followed with interest. As hypothesized, AaTAS1 is required for TeA biosynthesis, while AaMFS1 is an efflux pump for the transmembrane transport of TeA. Comparatively, the TeA yield of $\triangle A a T A S 1$ and $\triangle A a M F S 1$ dropped significantly compared with that of the wild-type strain. Specifically, the A domain of AaTAS1 catalyzed the start of TeA biosynthesis in vitro. Simultaneously, the pathogenicity of $\triangle A a T A S 1$ was also significantly decreased. Transcriptome analysis confirmed the abovementioned consistency between the TeA-producing phenotypes and related gene expression. Moreover, the proteins AaTAS1 and AaMFS1 were found present in the cytoplasm, plasma membrane, and intracellular membrane system, respectively, by fluorescence localization. Namely, AaTAS1 was responsible for the biosynthesis of TeA, and AaMFS1 was responsible for the efflux transport of TeA. Certainly, AaTAS1 indirectly regulated the expression of AaMFS1 through the level of synthetic TeA. Overall, data on the novel AaTAS1 and AaMFS1 genes mainly contribute to theoretical advances in mycotoxin biosynthesis and the pathogenicity of phytopathogens to agricultural foods.

${ }^{\dagger}$ Corresponding author: A. Wu; abwu@sibs.ac.cn

Sequencing data have been deposited in the National Center for Biotechnology Information database under BioProject PRJNA781145 (Run IDs: SRR16964232 to SRR16964237) and PRJNA781278 (Run IDs: SRR16970016 to SRR16970021) and BioProject PRJNA784742 (Run IDs: SAMN23500129 to SAMN23500134].

Funding: This work was funded by National Natural Science Foundation of China grant number U1703234 and NSFC-Distinguished Young Scholars of China grant number 32025030.

*The $\boldsymbol{e}$-Xtra logo stands for "electronic extra" and indicates there are supplementary materials published online.

The author(s) declare no conflict of interest.

(c) (1) () $\odot$ Copyright $(02022$ The Author(s). This is an open access article (c) ${ }_{\text {BY }}$ No No distributed under the CC BY-NC-ND 4.0 International license.
Keywords: AaMFS1, AaTAS1, biosynthesis, efflux transport, tenuazonic acid

Tenuazonic acid (TeA) is one of the most abundant metabolites in Alternaria alternata (Davis et al. 1977). Pure TeA is a brown oily substance that is slightly soluble in water and easily soluble in a variety of organic solvents, such as acetone, chloroform, ethanol, methanol, and ethyl acetate. TeA is very unstable, and it easily undergoes conformational changes under different $\mathrm{pH}$ values, different temperatures, and different solution medium conditions, to generate isomers such as isomer-TeA (Iso-TeA) (Lee et al. 2015) (Supplementary Fig. S1A). Therefore, it is generally converted into stable copper $\mathrm{Cu}(\mathrm{TeA})_{2}$ to save because TeA is a strong chelating agent. TeA shows low toxicity and low mutagenicity in vitro (Schwarz et al. 2012). However, it exhibits acute toxicity and synergistic effects with other mycotoxins in animals (Fraeyman et al. 2017; Puntscher et al. 2019). Mammals will suffer from dizziness, salivation, vomiting, tachycardia, visceral bleeding, circulatory failure, motor dysfunction, and even death after ingestion. The mechanism of TeA is to inhibit the formation of proteins in the ribosomes of eukaryotic cells (Aichinger et al. 2021).

TeA is a tetramic acid derivative produced by plant-pathogenic fungi, such as A. alternata, Pyricularia oryzae, and Epicoccum sorghinum (Motoyama et al. 2021; Oliveira et al. 2019). Experiments with labeled compounds in Alternaria tenuis have shown that the synthetic precursors of TeA are isoleucine and acetoacetylCoA (Stickings and Townsend 1961). However, the biosynthetic genes and synthetic pathways in A. alternata have not been resolved, so we can refer to the synthetic methods of TeA in other strains. Yun et al. (2015) identified the TeA biosynthesis gene TeA synthetase 1 (TAS1) in P. oryzae for the first time. TeA is synthesized by TAS1 from isoleucine and acetoacetyl-CoA. TAS1 is composed of nonribosomal peptide synthetase (NRPS) and polyketide synthase (PKS). It is a unique NRPS-PKS hybrid enzyme. TAS1 includes four domains, namely, adenylation (A), condensation (C), peptidyl carrier protein (PCP), and ketosynthase (KS). The biosynthesis process of TeA is as follows. First, L-isoleucine (L-Ile) is linked to the PCP under the catalysis of adenosylase domain $\mathrm{A}$; then, under the catalysis of the condensase domain $\mathrm{C}$, the amino group on L-Ile undergoes a condensation reaction with the internal carbonyl group of acetoacetyl-CoA to form an amide bond; and finally, with the help of the KS 
domain, it undergoes cyclization and is released to be product TeA (Yun et al. 2015).

After the biosynthetic pathway of TeA in P. oryzae, the team also discovered a zinc finger transcription factor encoding gene TAS2, which is located upstream of the TAS1 gene, and a global regulator, PoLAE1, which is a homolog of LaeA. Both $\triangle T A S 2$ and $\triangle P O L A E 1$ lose the ability to produce TeA under any form of induction. That validates the important role of the two genes in the regulation of TeA biosynthesis (Yun et al. 2017).

With the rapid development of fungal whole-genome sequencing, an increasing number of synthetic genes of secondary metabolites have been identified, including a large number of PKS and NPRS genes and clusters (Corre and Challis 2009; Kim et al. 2021; Scherlach and Hertweck 2021). These findings facilitate further studies of the biosynthesis process and regulation mechanism of TeA in A. alternata.

The major facilitator superfamily (MFS) is one of the largest membrane transporter families. These family members are widely distributed in bacteria, archaea, and eukaryotes and are closely related to many life activities (Yan 2015). Its basic role is to participate in the transport of substances across the membrane. MFS proteins can transport many small molecules, such as monosaccharides, polysaccharides, amino acids, peptides, vitamins, coenzyme factors, drug molecules, chromophores, and bases (Quistgaard et al. 2016). MFS superfamily proteins not only play an important role in the transport of many substances, but some of them are also closely related to immunological problems such as virus invasion and drug resistance in pathogenic organisms (Zhou et al. 2019).
Therefore, in this study, through homology comparison with other reference genes in key phytopathogens to main cereal crops, the target gene AaTAS1 in A. alternata was first found to likely be required for TeA biosynthesis. Then, near AaTAS1, AaMFS1 in A. alternata was of interest, probably acting as an efflux pump for TeA transmembrane transport. In detail, gene knockout and complement experiments were performed to accordingly confirm the functionalities and specific localization of these two target genes concerning the mycotoxin TeA production and pathogenicity of A. alternata as the main phytopathogens.

\section{RESULTS}

\section{Identification of AaTAS1 and discovery of AaMFS1.}

First, we found that CC77DRAFT_941807-encoding protein and TeA synthetase 1 (TAS1) in P. oryzae had $60.3 \%$ homology (Fig. 1A; Supplementary File S6). Moreover, based on de novo sequencing, we confirmed the existence of CC77DRAFT_941807 in the A. alternata XJaa strain. As a result, we renamed it AaTAS1. Analysis of the conserved domains on the Pfam website showed that AaTAS1 might be an NRPS-PKS hybrid enzyme containing four functional domains of $\mathrm{C}, \mathrm{A}, \mathrm{PCP}$, and $\mathrm{KS}$ (Fig. 1B; Supplementary File S7), so we speculate that the AaTAS1 gene is responsible for the biosynthesis of TeA.

In addition, we found that CC77DRAFT_269024 adjacent to AaTAS1 encoded 537 amino acids, had a conserved domain of MFS family transporters, and consisted of 14 transmembrane structures (Fig. 1B; Supplementary File S7). Consequently, we renamed it AaMFS1. In fungi, genes related to mycotoxin
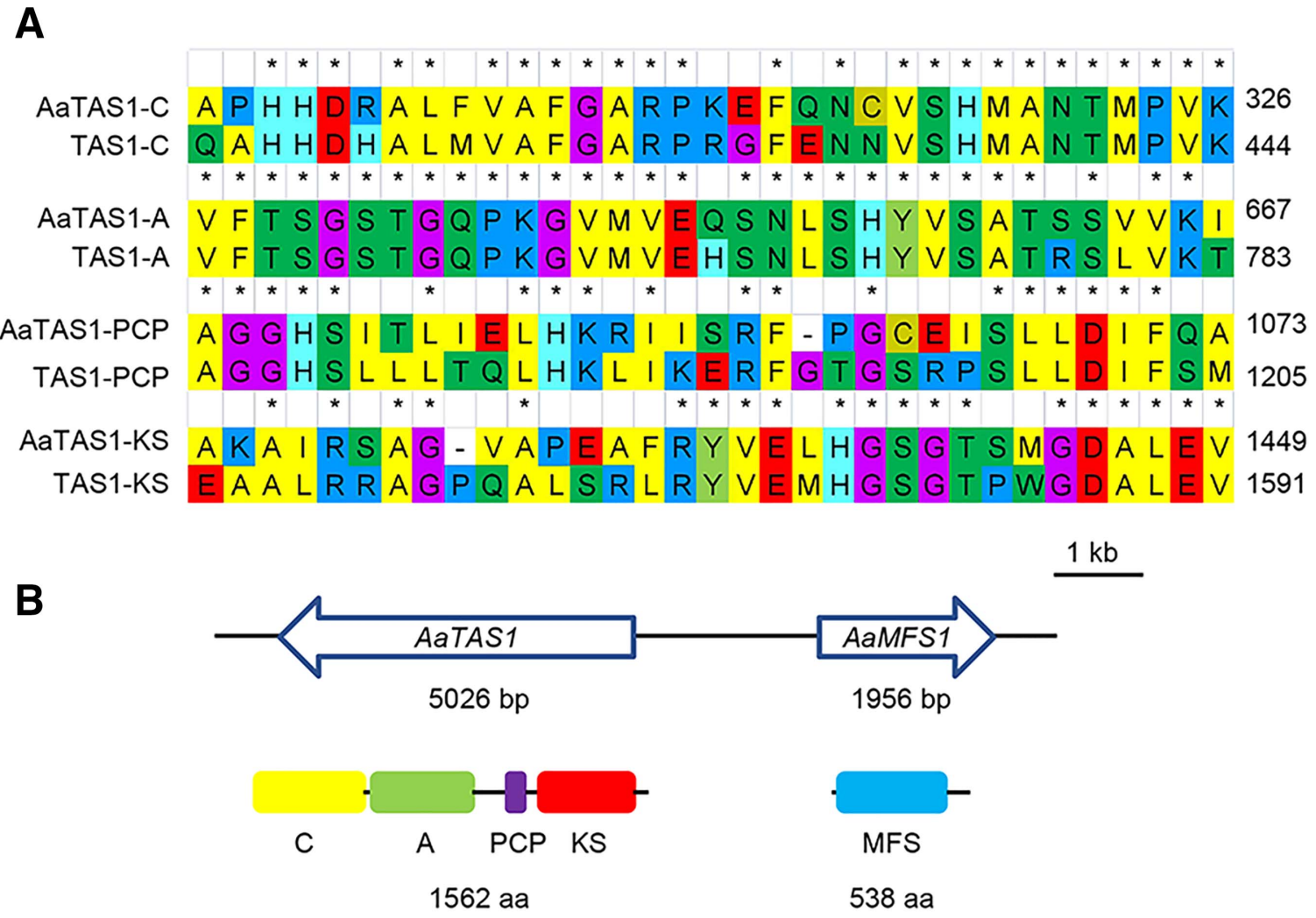

Fig. 1. Identification of AaTAS1 and AaMFS1. A, The amino acid sequences of AaTAS1 in Alternaria alternata and TAS1 in Pyricularia oryzae are homologously aligned in the sequences of condensation (C), adenylation (A), peptidyl carrier protein (PCP), and ketosynthase (KS). Different colors represent groups of amino acids with different properties. B, The gene CC77DRAFT_269024 was found upstream of CC77DRAFT_941807. The AaTAS1 protein is a nonribosomal peptide synthetase and polyketide synthase hybrid enzyme encoding 1,562 amino acids, with four domains of C, A, PCP, and KS; the AaMFS1 protein is a major facilitator superfamily (MFS) protein encoding 538 amino acids. The gene, mRNA, and protein sequence information for AaTAS1 and AaMFS1 can be found at the National Center for Biotechnology Information. Accession numbers are XP_018383119.1 and XP_018383120.1, respectively. 

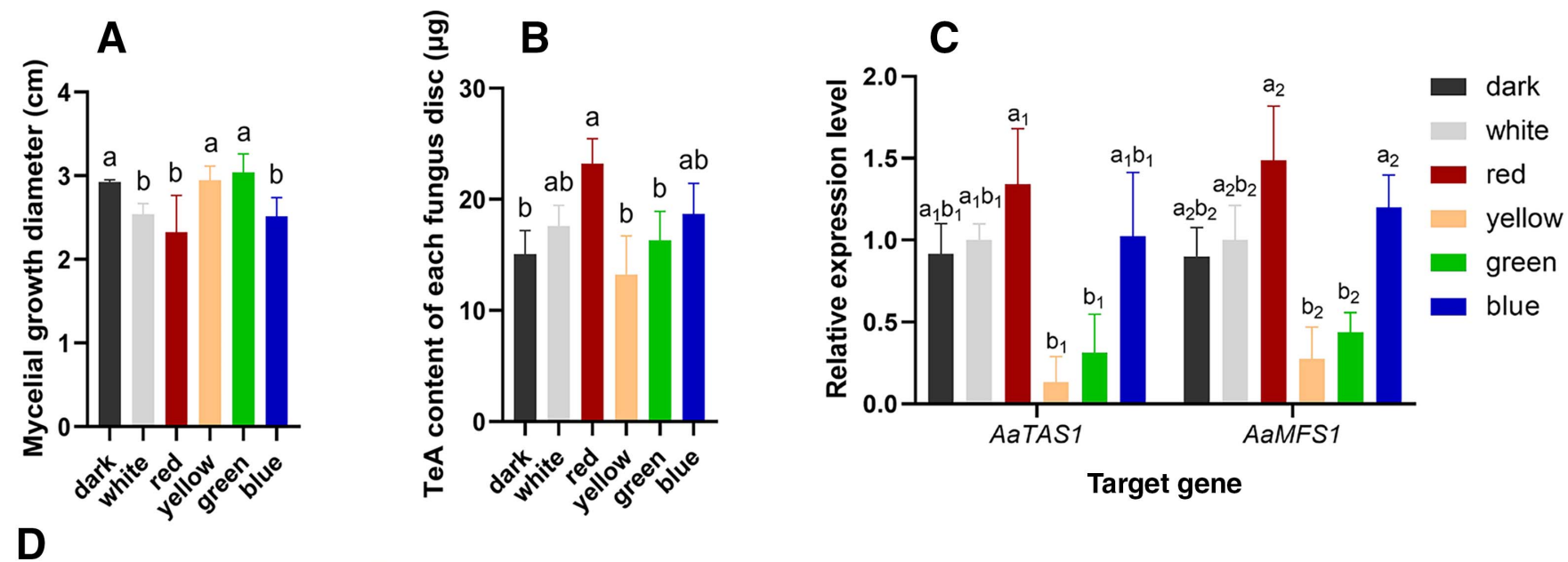

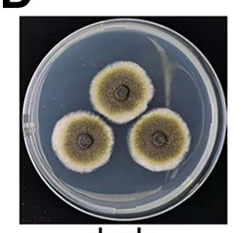

dark

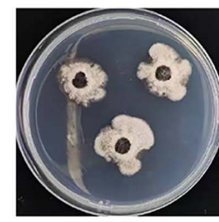

white

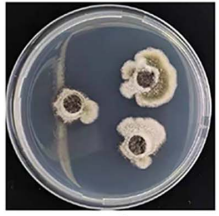

red

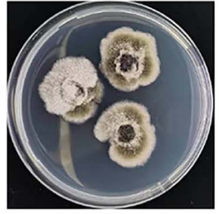

yellow

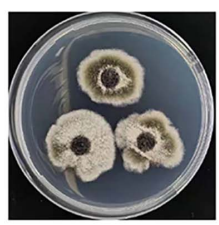

green

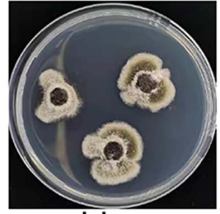

blue

Fig. 2. Effects of different light conditions on TeA production in Alternaria alternata. A, Effects of different light conditions on mycelial growth. B, Effects of different light conditions on TeA production. C, Effects of different light conditions on the expression of the AaTAS1 and AaMFS1 genes. D, The growth of A. alternata on potato dextrose agar under different light conditions was photographed on the third day postinoculation.
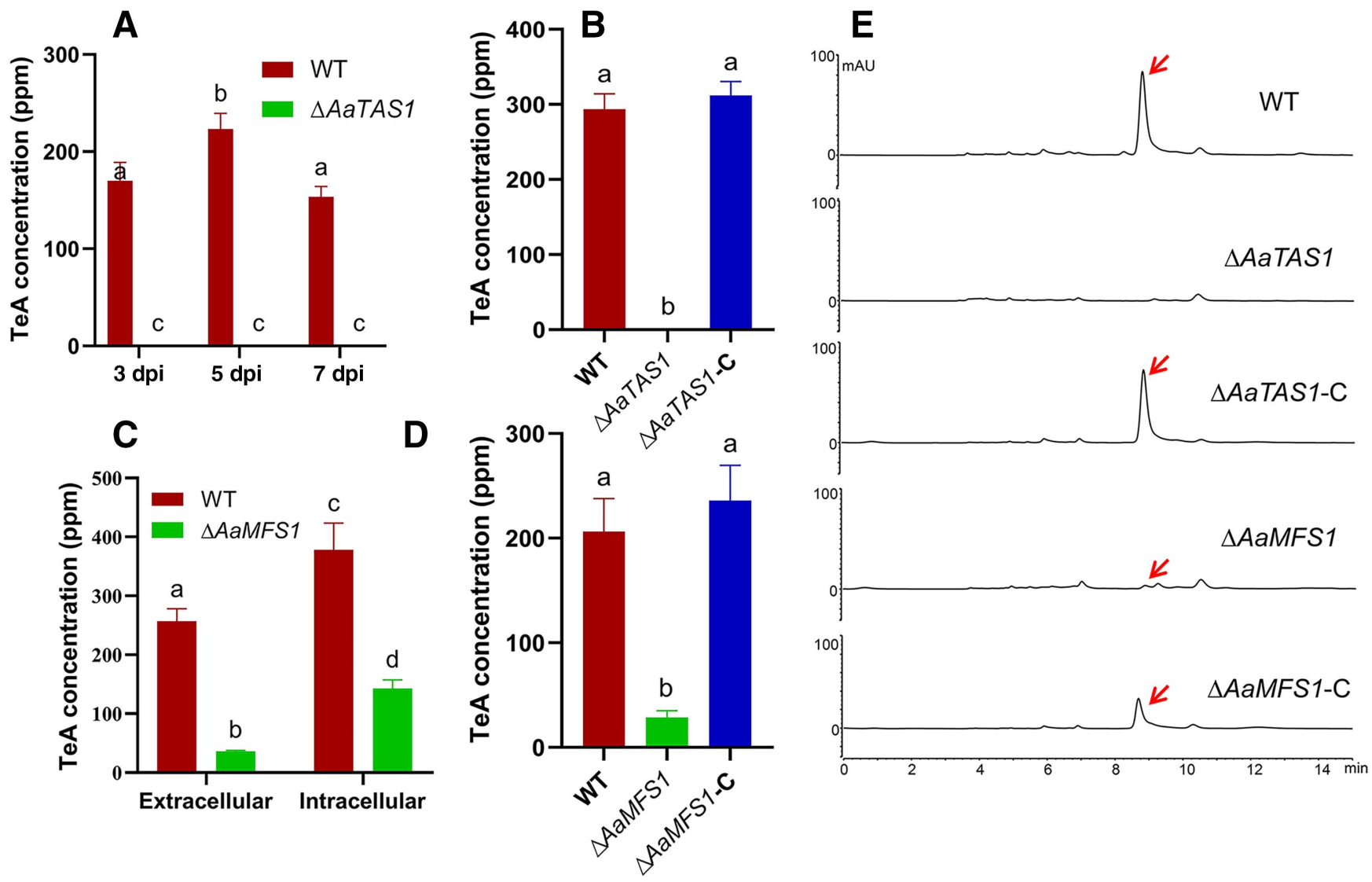

Fig. 3. Identification of TeA-producing phenotypes of different genotype strains. A, After knockout of the AaTAS1 gene, the production of TeA toxin could not be detected by ultraviolet high-pressure liquid chromatography (UV-HPLC) 3, 5, and 7 days postinoculation. B, The TeA yield of the $\triangle A a T A S 1$ and $\triangle A a T A S 1-C$ strains in liquid medium. The knockout strain could not produce TeA, and the TeA-producing phenotype of the complement strain was recovered. C, Compared with the wild type, the extracellular and intracellular TeA yield of the $\triangle$ AaMFS1 strain was lower. D, The TeA yield of the $\triangle A a M F S 1$ strain in liquid medium decreased significantly, and the TeA yield of the $\triangle A a M F S 1-\mathrm{C}$ strain returned to the wild-type level. E, UV-HPLC chromatograms of gene knockout and complementary strains. 
synthesis and metabolism are usually clustered (Bills and Gloer 2016). Therefore, we speculated that AaMFS1 might be involved in the transport of TeA.

\section{Correlation between the toxigenic phenotype and} AaTAS1 and AaMFS1 gene expression.

Under different environmental conditions, TeA production by A. alternata will also be affected (Stinson 1985). We tested the influence of different environmental conditions on TeA production and detected the relative expression levels of the two genes AaTAS1 and AaMFS1 to confirm whether the two genes corresponded to the TeA production phenotype.

We tested the effects of different light colors on A. alternata. The white light was separated into different colors by color filters, and $A$. alternata cultivated on potato dextrose agar (PDA) plates was irradiated. The results showed that red light had a relatively obvious inhibitory effect on the growth of A. alternata (Fig. 2A and D) and, under red light illumination, TeA production by
A. alternata also increased to a certain extent. In contrast, under irradiation with yellow and green light, the production of TeA was low (Fig. 2B). The relative expression levels of the AaTAS1 and AaMFS1 genes were highest under red and lowest under yellow and green light conditions (Fig. 2C), which was compatible with the phenotype of increased TeA production.

\section{Genetic evidence confirms the association between the AaTAS1 and AaMFS1 genes and TeA production.}

We used the A. alternata XJaa strain isolated from jujube as the research material to knock out AaTAS1 (Supplementary Fig. S2A). Knockout of AaTAS1 resulted in the inability to synthesize TeA (Fig. 3A and E), which indicated that AaTAS1 was a synthetase of $\mathrm{TeA}$ in A. alternata. To verify this conclusion, we constructed an AaTAS1 gene-complemented mutant strain, $\triangle A a T A S 1-\mathrm{C}$, and found that the TeA synthesis ability of the complemented mutant strain was restored (Fig. 3B and E), further confirming the ability of the AaTAS1 gene to control TeA biosynthesis.

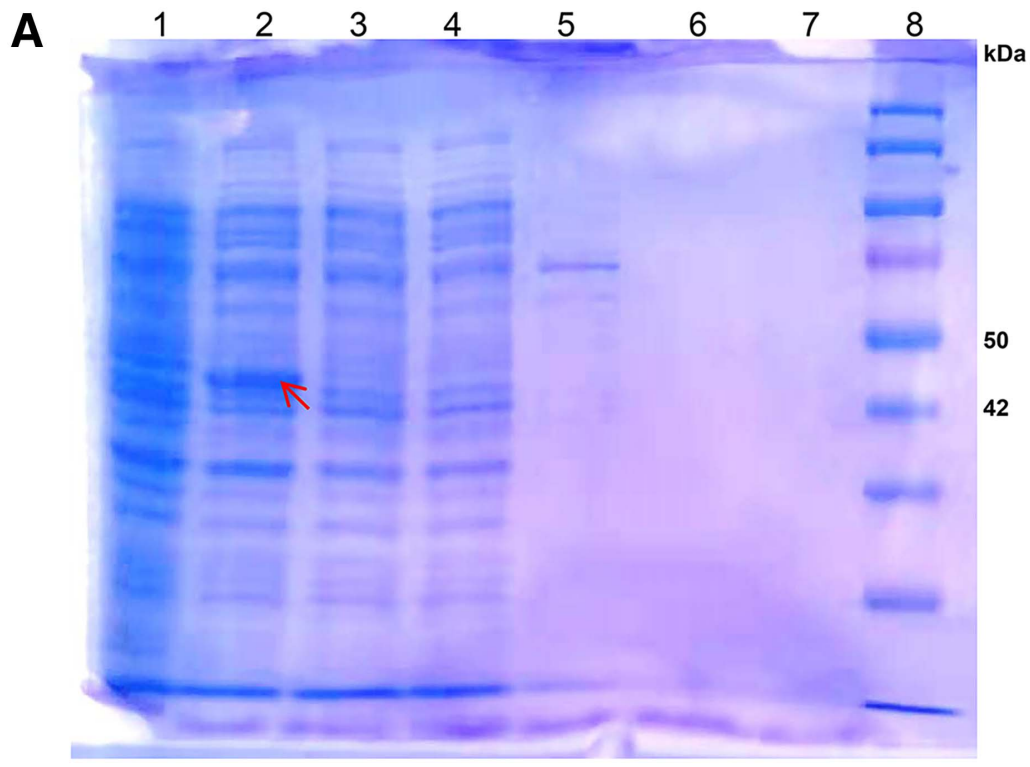

\section{C}

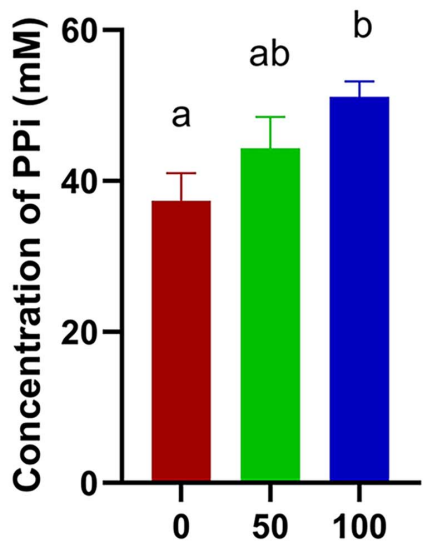

\section{Protein Concentration $(\mu \mathrm{g} / \mathrm{ml})$}

B

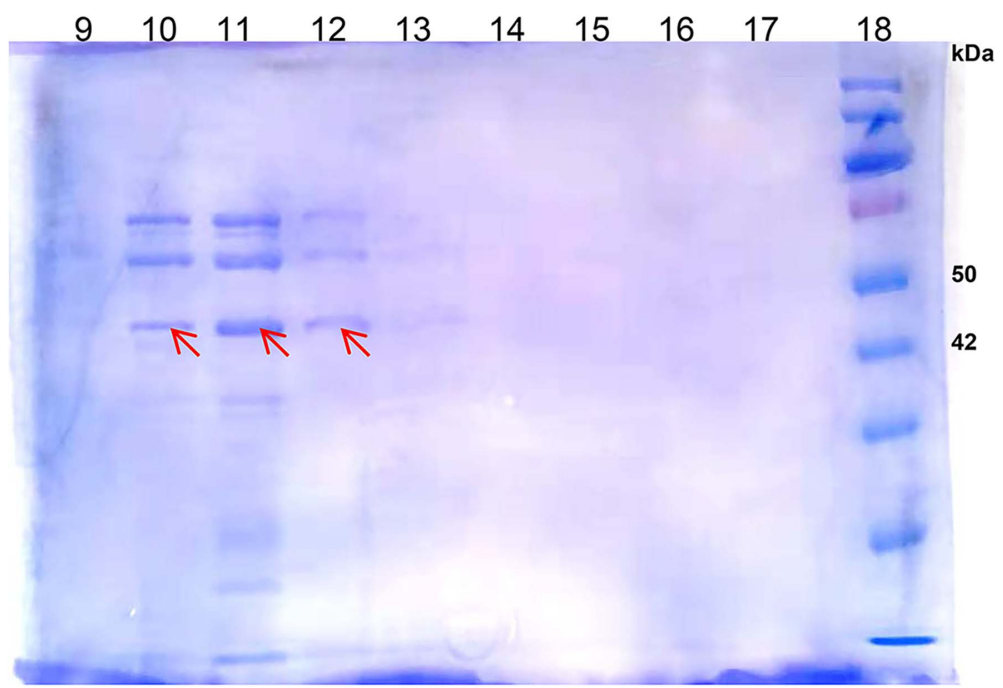

D

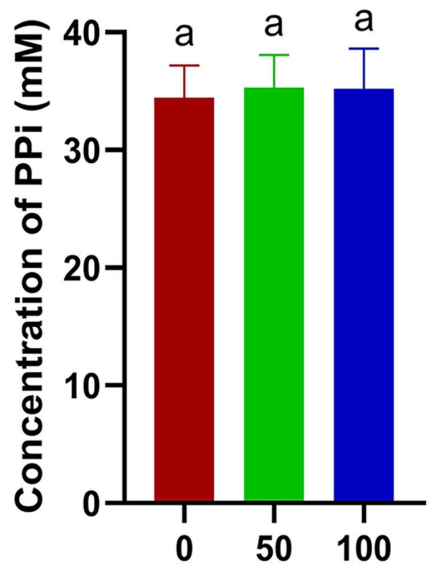

\section{Denatured Protein Concentration $(\mu \mathrm{g} / \mathrm{ml})$}

Fig. 4. Functional verification of the AaTAS1-A domain. A and B, Sodium dodecyl sulfate-polyacrylamide gel electrophoresis of protein solutions in different steps. Lane 1: The lysate of BL21 not induced by isopropyl- $\beta$-D-thiogalactoside (IPTG); lane 2 : the lysate of BL21 cell induced by IPTG; lane 3: supernatant after centrifugation; lane 4: loading effluent passed through a nickel column; lanes 5 to 7: washing effluents; lane 8: protein marker; and lanes 9 to 17: elution effluents in different periods. The molecular weight of the target protein AaTAS1-A-His is approximately $48 \mathrm{kDa}$. $\mathbf{C}$, In the reaction system with different protein concentrations, a pyrophosphate (PPi) detection kit was used to measure the production of PPi. $\mathbf{D}$, After denaturing the protein at $100^{\circ} \mathrm{C}$ for 15 min, the test was repeated to determine the production of PPi. 
Knockout of AaMFS1 resulted in a significant decrease in the extracellular TeA concentration. However, in contrast to our expectations, while the extracellular TeA content decreased, the intracellular TeA content also decreased (Fig. 3C and E). We speculate that is because the overall TeA production level of $A$. alternata had decreased in $\triangle A a T A S 1$. In addition, the extracellular TeA concentration of $\triangle A a M F S 1-\mathrm{C}$ returned to the wild- type level (Fig. 3D and E). The above results indicate that AaMFS1 is likely to be the efflux pump for mycotoxin TeA from intracellular to extracellular.

\section{Functional verification of AaTAS1-A domain.}

The AaTAS1-A domain is responsible for the first step of the biosynthesis of $\mathrm{TeA}$, and there is no need to synthesize

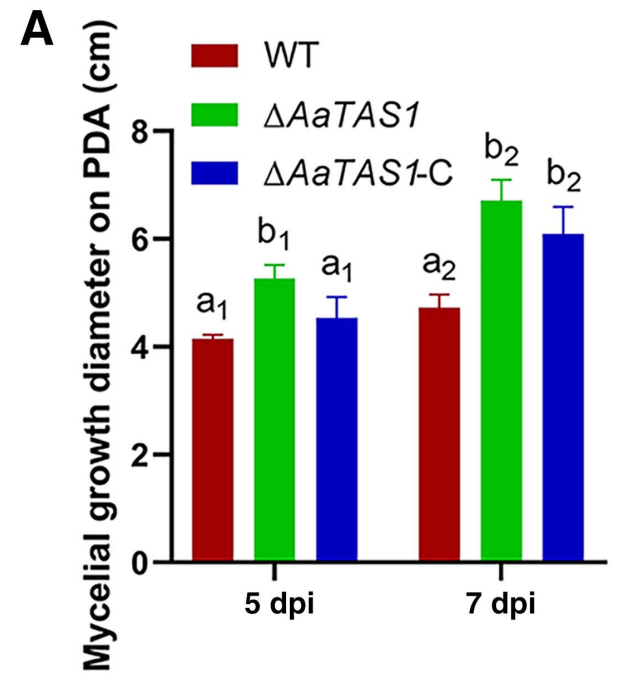

B
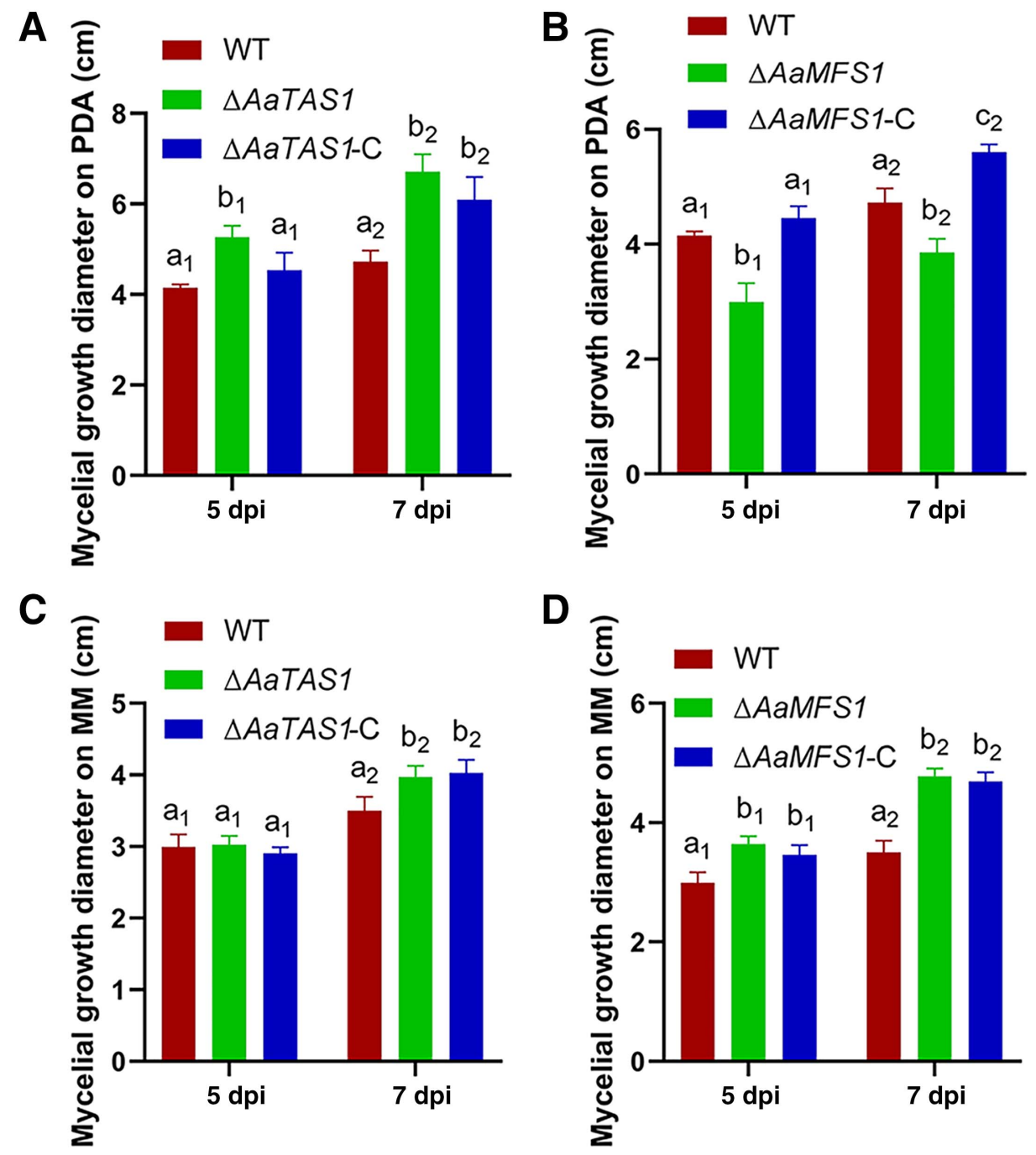

D
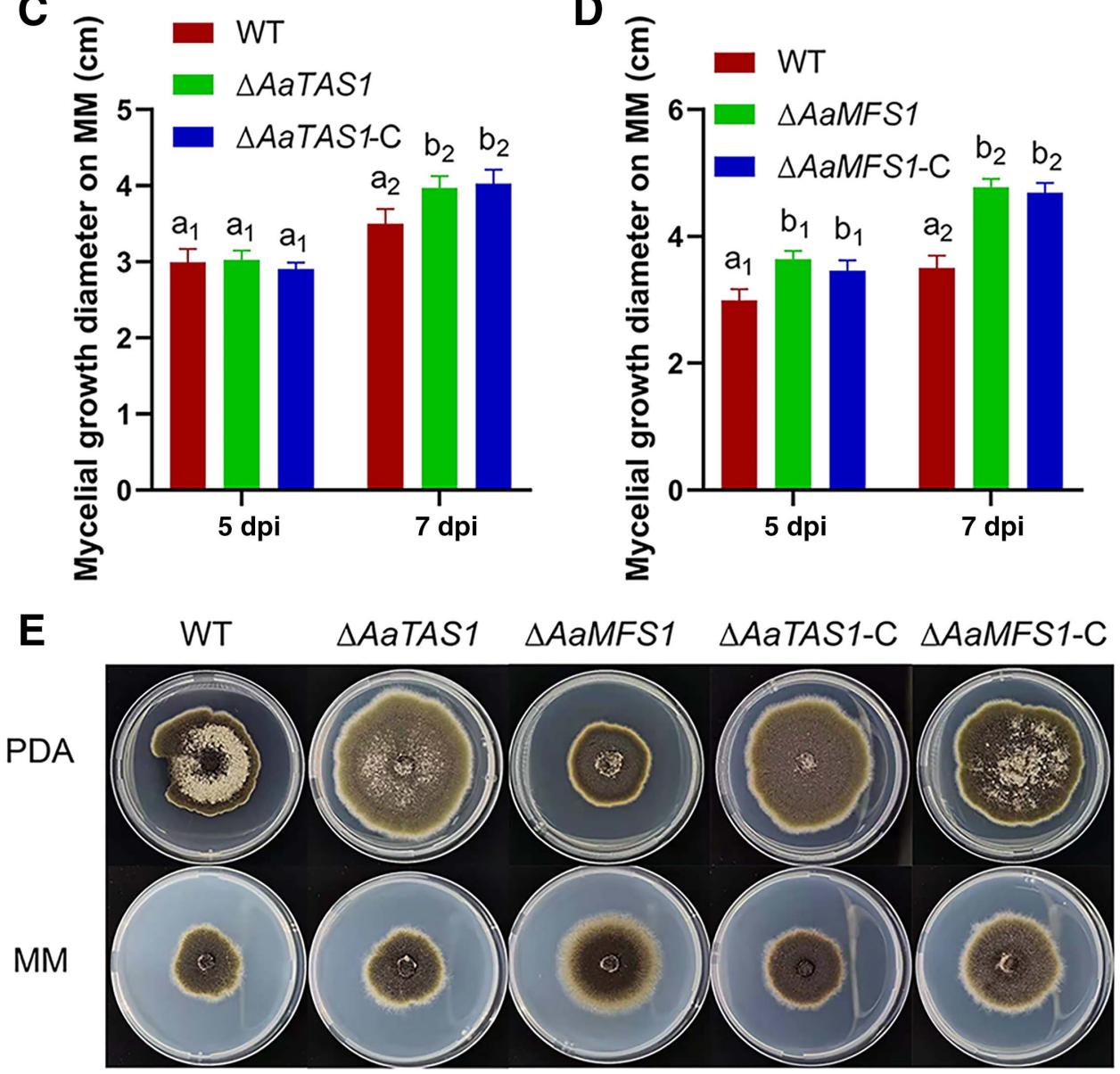

Fig. 5. Growth of AaTAS1 and AaMFS1 gene knockout strains and complementary strains on potato dextrose agar (PDA) and minimal medium (MM) plates. A, The growth diameter of the wild-type, $\triangle A a T A S 1$, and $\triangle A a T A S 1-\mathrm{C}$ strains on PDA at 5 and 7 days postinoculation (dpi). B, The growth diameter of wild-type, $\triangle A a M F S 1$, and $\triangle A a M F S 1-\mathrm{C}$ strains on PDA at 5 and $7 \mathrm{dpi}$. C, The growth diameters of wild-type, $\triangle A a T A S 1$, and $\triangle A a T A S 1$-C strains on MM medium at 5 and $7 \mathrm{dpi}$. D, The growth diameters of wild-type, $\triangle A a M F S 1$, and $\triangle A a M F S 1$-C strains on MM medium at 5 and 7 dpi. E, The growth of different genotype strains on PDA and MM was photographed 7 dpi. 
intermediate for experiment. As a result, we chose AaTAS1-A to verify its function. We expressed the AaTAS1-A domain heterologously in Escherichia coli and then disrupted the bacterial cells by ultrasonication. We removed cell debris by centrifugation and obtained the purified protein from the supernatant through a nickel column. We used the protein concentrate to construct reaction systems with different protein concentrations (Fig. 4A and B).

According to domain prediction, we know that the basic function of AaTAS1-A is to catalyze the reaction of L-Ile and adenosine triphosphate (ATP) to produce pyrophosphate (PPi) and Ile-adenosine monophosphate (Ile-AMP) (Stanišić and Kries 2019). Therefore, we can demonstrate that the reaction process has taken place simply by measuring the change in the product PPi using a PPi detection kit.
The results showed that when the protein concentration reached $100 \mu \mathrm{g} / \mathrm{ml}$, the content of PPi in the reaction system was significantly different from that in the control group without protein (Fig. 4C), which confirmed that the AaTAS1-A domain had the ability to catalyze this reaction. To ensure the accuracy of the results, we also boiled the protein at $100^{\circ} \mathrm{C}$ for $15 \mathrm{~min}$ to completely denature it. We found that the protein lost its catalytic ability after denaturation (Fig. 4D).

\section{Knockout of AaMFS1 slows down the growth rate of A. alternata on PDA.}

Knockout of AaTAS1 did not inhibit the growth of A. alternata. In contrast, the growth diameter of $\triangle A a T A S 1$ was slightly higher than that of the wild-type strain (Fig. 5A and E). However, the growth rate of $\triangle A a M F S 1$ on PDA was significantly
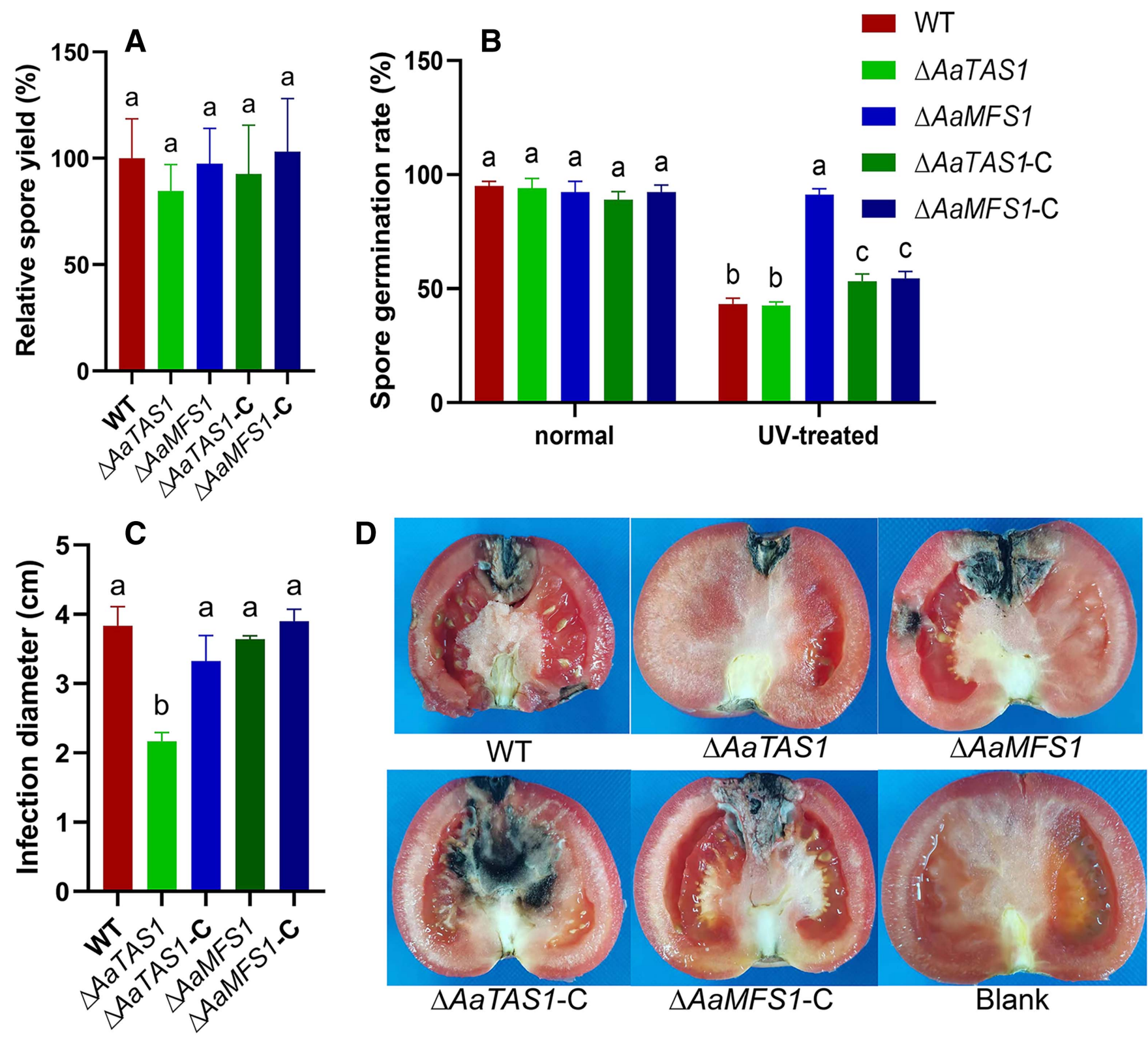

Fig. 6. Effects of AaTAS1 and AaMFS1 on the spore germination, UV resistance, and pathogenicity of Alternaria alternata. A, Relative spore yield of wild-type, $\triangle A a T A S 1$, and $\triangle A a T A S 1-\mathrm{C}$ strains, $\triangle A a M F S 1$ and $\triangle A a M F S 1-\mathrm{C}$ strains on potato dextrose agar plates. B, The spore germination rates of wild-type, $\triangle$ AaTAS1, and $\triangle A a T A S 1-\mathrm{C}$ strains, $\triangle A a M F S 1$ and $\triangle A a M F S 1-\mathrm{C}$ strains, and the changes in spore germination rate after UV irradiation for $30 \mathrm{~min}$. C, For the wild-type, $\triangle A a T A S 1$, and $\triangle A a T A S 1-\mathrm{C}$ strains and the $\triangle A a M F S 1$ and $\triangle A a M F S 1$-C strains, we inoculated fungal discs on the wounds on top of tomatoes and measured the diameter of disease spots 8 days postinoculation (dpi). D, The concentration of quantitative spore suspension was $10^{7}$ per milliliter. We added $10 \mu \mathrm{l}$ of the spore solution to the wound on the top of tomatoes and cut it at 8 dpi to observe the $A$. alternata infection in tomatoes. 
slower than that of the wild type (Fig. 5B and E). This result indicated that the AaMFS1 gene could not only function as an efflux pump of TeA but also affect other pathways, thereby promoting A. alternata growth. Interestingly, we also measured the growth diameters of different genotype strains on minimal medium (MM) plates, and there was no significant change in $\triangle A a T A S 1$ and $\triangle A a M F S 1$ compared with the wild type (Fig. 5C, D, and E).

\section{Knockout of AaMFS1 improves the tolerance of $A$. alternata to $\mathrm{UV}$.}

By scraping from the surface of the A. alternata colony grown on PDA, we compared the relative spore yields of different genotype strains. The results indicated that the AaTAS1 and AaMFS1 genes had no significant effect on the sporulation ability of A. alternata (Fig. 6A).

We evenly spread the spore suspension obtained in the previous step on a water agar plate. After culturing at $25^{\circ} \mathrm{C}$ for approximately $8 \mathrm{~h}$, we counted the germination rate of the spores under an inverted microscope. We found that there was no significant difference between different genotype strains and the germination rate was approximately $90 \%$ (Fig. 6B).

On the basis of this experiment, we wanted to explore whether there was any change in the UV resistance level of A. alternata. After spreading, the lid of the Petri dish was opened in the ultraclean workbench for UV irradiation and, after incubation, the spore germination rate of each group was counted again. The results showed that the spore germination rate in all groups except $\triangle A a M F S 1$ was reduced to 40 to $50 \%$ (Fig. $6 \mathrm{~B}$ ).

\section{AaTAS1 contributes to the pathogenicity of A. alternata.}

A. alternata was inoculated on healthy tomatoes to form infection spots to measure the changes in pathogenicity. Experimental evidence showed that after the AaTAS1 gene was knocked out, the diameter of the lesion circle was significantly reduced. However, after the AaTAS1 gene was complemented, its virulence to tomatoes was restored (Fig. 6C and D). In the $\triangle A a M F S 1$ strain, the lesion diameter did not change significantly, indicating that the AaMFS1 gene was not necessary for pathogenicity (Fig. 6C and D).

\section{Transcriptome analysis unravels the global influence of AaTAS1 and AaMFS1.}

Compared with the wild type, the $\triangle$ AaTAS1 strain had 199 upregulated and 199 downregulated differential genes and the $\triangle A a M F S 1$ strain had 1,345 upregulated and 843 downregulated differential genes (Supplementary Fig. S4). AaMFS1 showed a wider influence than AaTAS1.

Kyoto Encyclopedia of Genes and Genomes (KEGG) pathway enrichment analysis was performed on the differentially expressed genes of the two groups. The results showed that AaTAS1 and AaMFS1 might participate in many metabolic
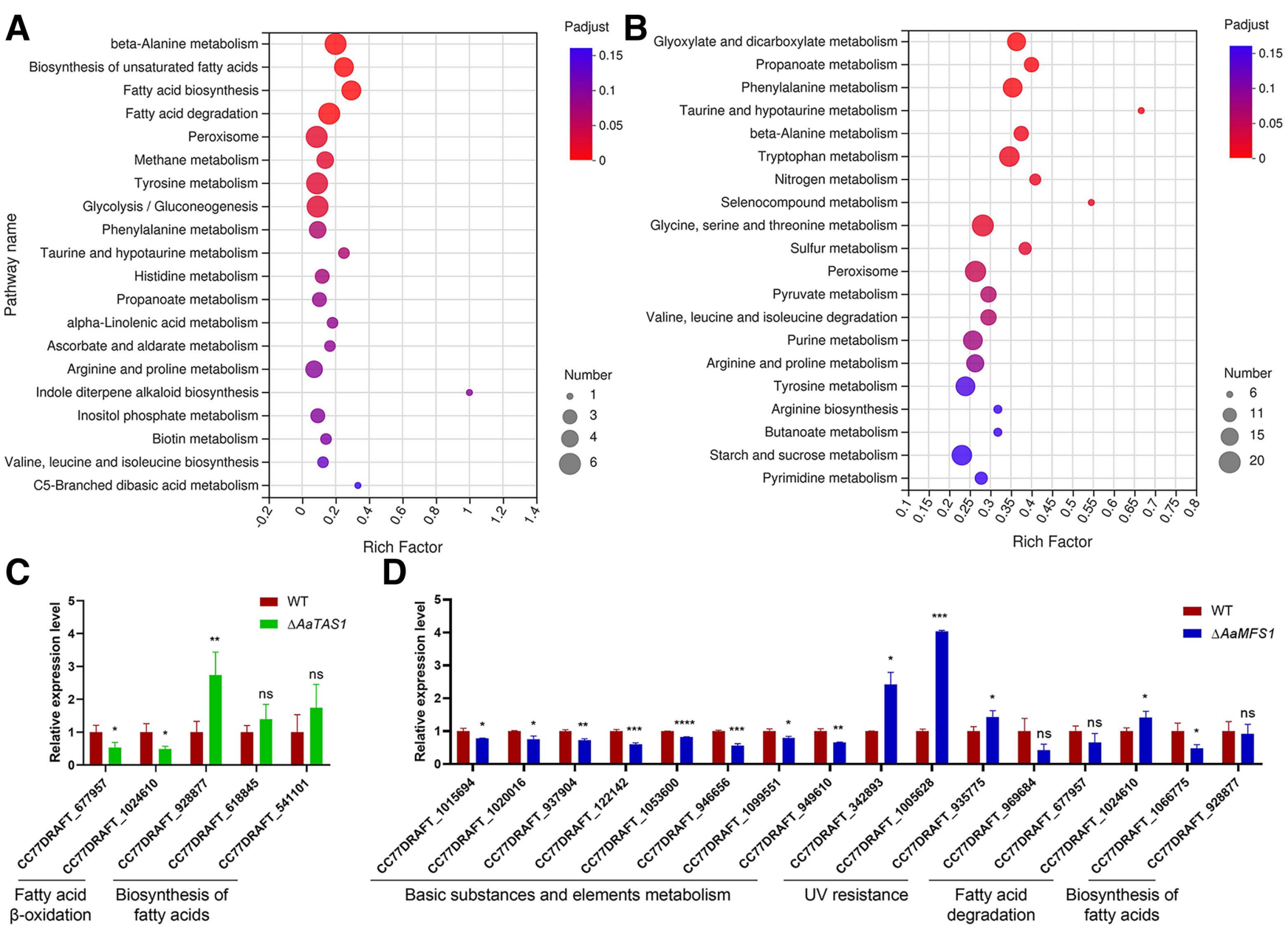

D

Fig. 7. Transcriptome analysis unravels the global influence of AaTAS1 and AaMFS1. A, Kyoto Encyclopedia of Genes and Genomes (KEGG) enrichment analysis of transcriptome differential genes in the $\triangle A a T A S 1$ strain. B, KEGG enrichment analysis of transcriptome differential genes in the $\triangle A a M F S 1$ strain. C, Verification of the expression level of key genes in the fatty acid metabolism pathway of the $\triangle A a T A S 1$ strain. D, Verification of key gene expression levels in basic substance and element metabolism and UV resistance-related and fatty acid metabolism pathways of the $\triangle A a M F S 1$ strain. 
pathways. The differentially expressed genes of $\triangle$ AaTAS1 were mainly enriched in fatty acid synthesis and degradation, peroxisome-related pathways, glycolysis and gluconeogenesis, and various amino acid metabolism and other pathways (Fig. 7A). The differentially expressed genes of $\triangle A a M F S 1$ were mainly enriched in the metabolism of small molecular organic acids, such as glyoxylate, dicarboxylate, propanoate, pyruvate and various amino acid metabolism pathways, the metabolism of basic elements, such as sulfur and nitrogen, and the metabolism of basic substances, such as purine and pyrimidine and other pathways (Fig. 7B).

To match the phenotypic changes of knockout strains found previously, we selected some genes for verification by reverse transcription-quantitative PCR (RT-qPCR). The expression levels of these genes have already been confirmed to change significantly in the transcriptome (Supplementary Tables S11 and S12). In $\triangle A a T A S 1$, we chose some genes involved in fatty acid oxidative decomposition and synthesis pathways. These results showed that, while knockout of AaTAS1 blocked the synthesis of TeA toxin, it also disrupted the expression of some genes involved in fatty acid metabolism (Fig. 7C). In $\triangle A a M F S 1$, we mainly chose some genes involved in the pathways of basic substance and element metabolism, UV resistance, and fatty acid degradation and synthesis. CC77DRAFT_342893 and CC77DRAFT_1005628 are both related to nucleotide-excision repair, which is responsible for repairing DNA damage caused by UV (Kumar et al. 2020; Sinha and Häder 2002). The CC77DRAFT_935775-coding protein Yap1 is a DNA damage responder found in Saccharomyces cerevisiae (Rowe et al. 2012). These results showed that the basic substance and element metabolism-related genes in $\triangle A a M F S 1$ were generally downregulated, and several genes related to UV resistance were significantly upregulated. Similar to $\triangle A a T A S 1$, the expression level of some genes involved in fatty acid metabolism changed significantly (Fig. 7D).

\section{The location of AaTAS1 and AaMFS1 proteins.}

The plasmid carrying AaTAS1-GFP (green fluorescent protein) and AaMFS1-GFP was integrated into the A. alternata genome by random insertion (Supplementary Fig. S2B). We extracted the genome of transformants for the amplification of hygromycin characteristic fragments, thus confirming the existence of positive transformants (Supplementary Fig. S2D). We picked the marginal hyphae of transformants to make slides and confirmed that AaTAS1-GFP and AaMFS1-GFP could be expressed in both hyphae and spores (Fig. 8A).
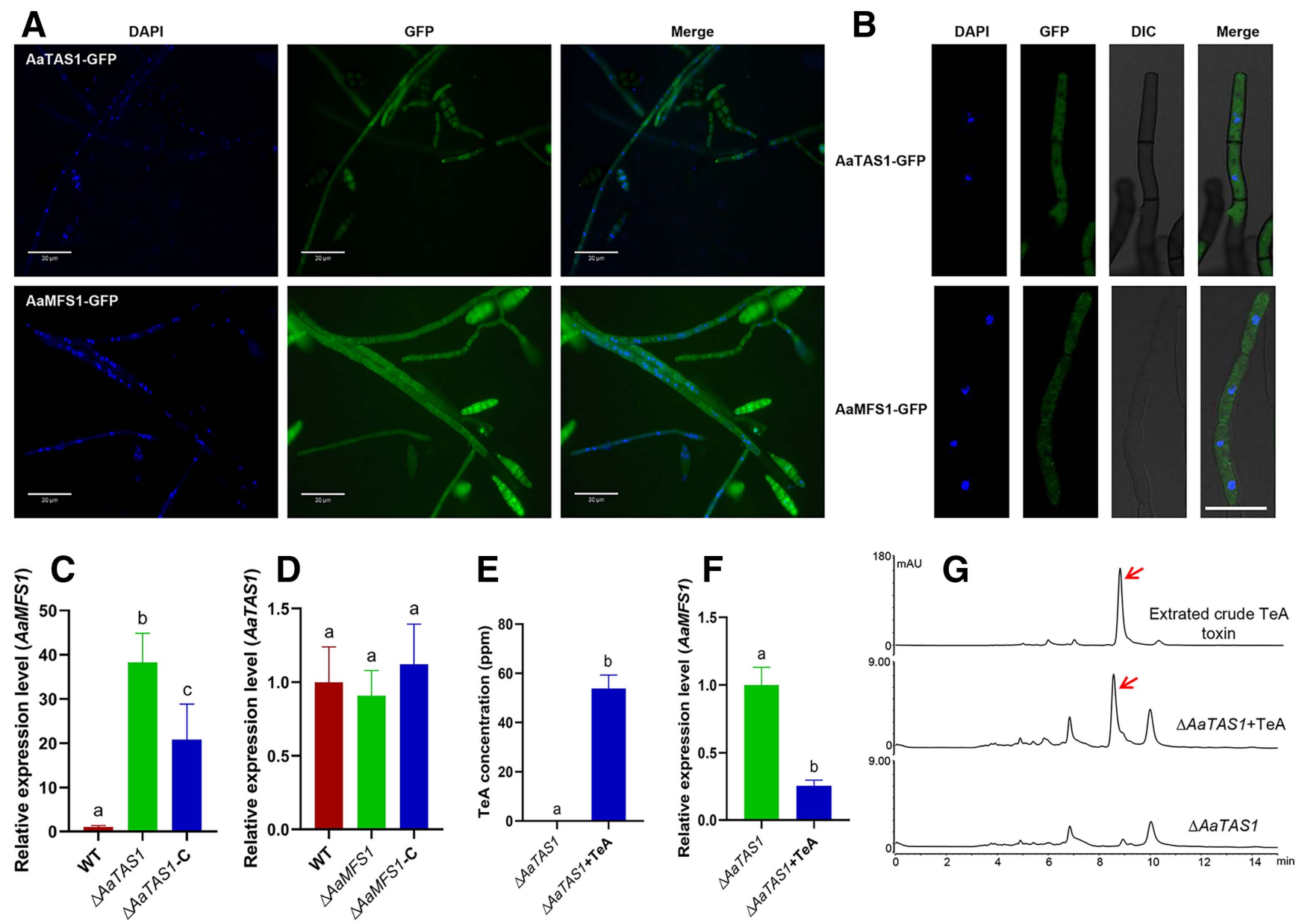

Fig. 8. Localization and interaction of AaTAS1 and AaMFS1. A, Localization of AaTAS1-GFP and AaMFS1-GFP fluorescent proteins in hyphae and spores. 4',6-Diamidino-2-phenylindole and green fluorescent protein (GFP) were photographed, which showed that the two proteins could be expressed in both mycelia and spores. Scale bar $=30 \mu \mathrm{m}$. B, Localization of AaTAS1-GFP and AaMFS1-GFP proteins in cells photographed by confocal fluorescence microscopy. Scale bar $=20 \mu \mathrm{m}$. C, The expression level of the AaMFS1 gene in the wild-type, $\triangle A a T A S 1$, and $\triangle A a T A S 1$-C strains. D, The expression level of the AaTAS1 gene in the wild type, $\triangle A a M F S 1$, and $\triangle A a M F S 1-\mathrm{C}$ strains. E, The TeA crude extract was added to the liquid medium of the $\triangle A a T A S 1$ strain to approximately $60 \mathrm{ppm}$. On the third day postinoculation, the TeA content in the culture medium was measured again. F, The relative expression of the AaMFS1 gene in the $\triangle A a T A S 1$ strain grown in PDB with TeA and potato dextrose broth without TeA. G, Ultraviolet high-pressure liquid chromatography chromatograms of TeA crude extract, the TeA addition group, and the control group. 
By fluorescence confocal microscopy, we confirmed the subcellular localization of the two proteins. AaTAS1 was mainly distributed in the cytoplasm rather than the vacuoles in hyphal cells. AaMFS1 was mainly distributed on the plasma membrane and intracellular membrane system, especially the plasma membrane at the junction of two hyphal cells (Fig. 8B).

\section{Indirect interaction between AaTAS1 and AaMFS1 proteins.}

In the $\triangle A a T A S 1$ strain, AaMFS1 gene expression was significantly upregulated (Fig. 8C). However, in the $\triangle A a M F S 1$ strain, AaTAS1 gene expression did not change significantly (Fig. 8D). In summary, we hypothesize that there may be a direct or indirect interaction between AaTAS1 and AaMFS1.

Because AaMFS1 is located on the membrane, the yeast twohybrid method cannot be used to test the interaction of AaMFS1 and AaTAS1. We also attempted to construct the pDL2 plasmid carrying AaTAS1-GFP and the pFL7 plasmid carrying AaMFS1-Flag and transformed them into XJaa simultaneously for the immunoprecipitation experiments. However, we were not able to successfully extract the AaMFS1-Flag protein. We hypothesized that this might be because AaMFS1 is a transmembrane protein. Therefore, we could only choose some indirect methods to test the interaction between the two proteins.

We hypothesized that AaTAS1 and AaMFS1 may have an indirect interaction through TeA. Therefore, we prepared TeA crude extracts (Fig. 8G) to add to the liquid medium of $\triangle A a T A S 1$ and ensured that the final concentration of TeA reached $60 \mathrm{ppm}$. At 3 days postinoculation (dpi), the concentration of TeA in the potato dextrose broth (PDB) was measured again to confirm that it had not been broken down (Fig. 8E and G). The results showed that the presence of TeA could reduce the expression level of the AaMFS1 gene. In other words, TeA inhibited the expression of the AaMFSI gene (Fig. 8F).

\section{DISCUSSION}

We found that the protein encoded by CC77DRAFT_941807 showed high homology with the TeA biosynthetic enzyme
TAS1 in $P$. oryzae. In addition, through de novo sequencing, we obtained the candidate gene AaTAS1 for mycotoxin TeA biosynthesis. Later, the MFS transporter gene AaMFS1 was found upstream of the AaTAS1 gene. We hypothesize that AaTAS1 is responsible for the synthesis of TeA and that AaMFS1 is responsible for the transport of $\mathrm{TeA}$ across the membrane (Fig. 9).

First, to confirm that the two genes AaTAS1 and AaMFS1 were related to the biosynthesis and metabolism of TeA, we explored the correlation between the changes in TeA production and the expression levels of the two genes. Under unfavorable environments, fungi tend to produce mycotoxins, thereby increasing their adaptability to unfavorable environments and their ability to parasitize the host (Fu et al. 2020). For example, under irradiation with red light, the growth of A. alternata was inhibited. Concurrently, the yield of TeA was also significantly increased, and the expression levels of the AaTAS1 and AaMFS1 genes were simultaneously increased. These results indicate that AaTAS1 and AaMFS1 may be involved in the biosynthesis and metabolic regulation of TeA.

Furthermore, reverse genetic experiments validated that, after the AaTAS1 gene was knocked out, $\triangle A a T A S 1$ lost the ability to synthesize TeA; after the AaMFS1 gene was knocked out, the extracellular TeA level of $\triangle A a M F S 1$ decreased, consistent with our expectations.

We also wanted to use biochemical evidence to confirm the TeA synthesis ability of the AaTAS1 protein. We chose the AaTAS1-A domain to verify its function. We expressed the AaTAS1-A protein through an E. coli heterologous expression system, harvested and purified the protein (Scopes 2001), and constructed reaction systems with different protein concentrations. Compared with the base value of the protein-free control group, the amount of PPi produced by the high-concentration protein group was significantly increased, confirming the ability of the AaTAS1-A domain to catalyze the reaction from L-Ile and ATP to PPi and Ile-AMP.

Moreover, the knockout strains also showed phenotypes other than TeA production. The growth rate of $\triangle A a T A S 1$ on PDA was slightly increased, and the growth rate of $\triangle A a M F S 1$ on PDA was significantly reduced. However, on MM plates, the

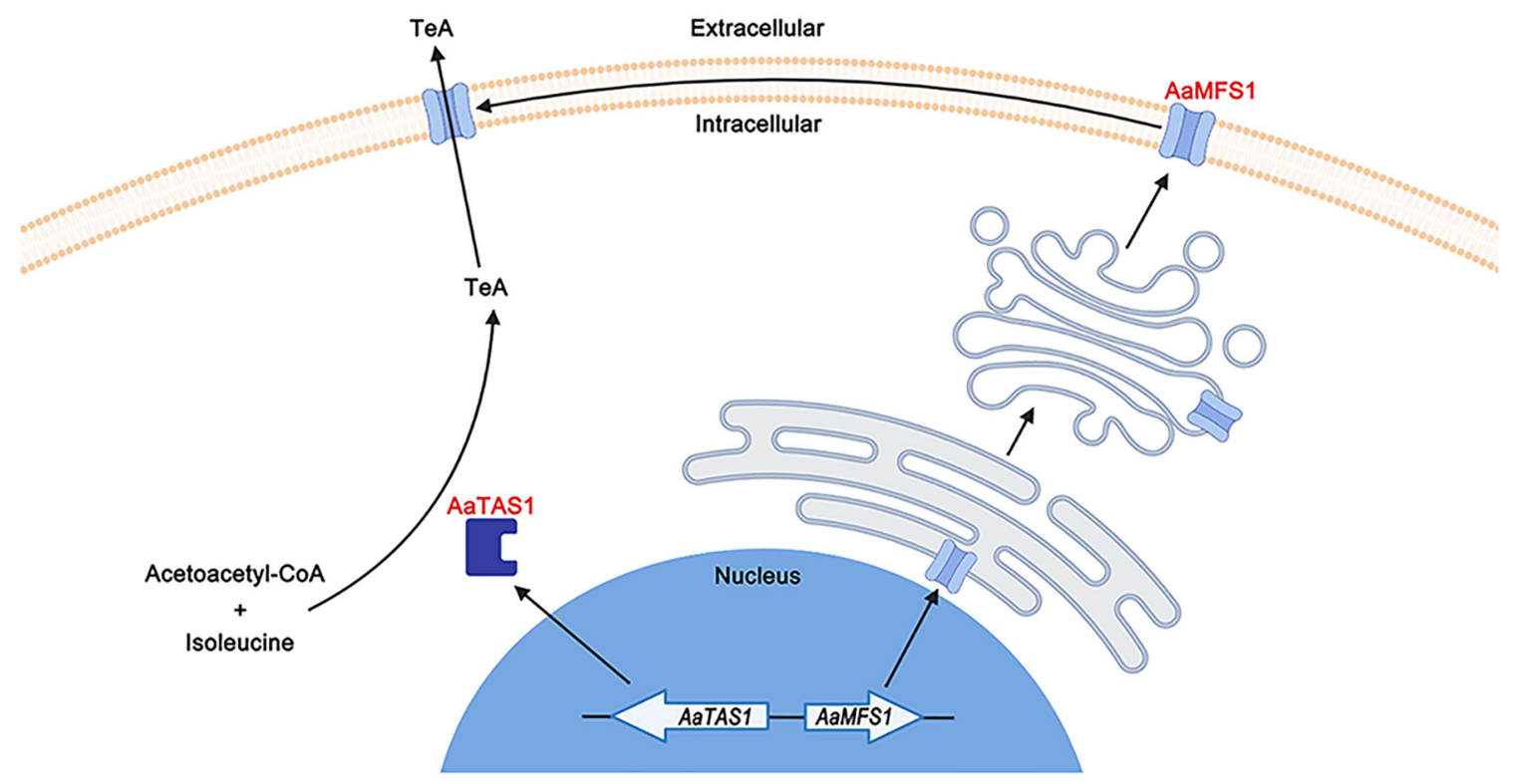

Fig. 9. Working model of AaTAS1 and AaMFS1 in cells. The protein encoded by the AaTAS1 gene is a TeA biosynthetic enzyme, which is distributed in the cytoplasm and participates in the reaction of consuming isoleucine and acetoacetyl-CoA to produce TeA. AaMFS1 encodes a TeA transporter that is distributed in the plasma membrane and cell endomembrane system. The AaMFS1 protein is responsible for the intracellular to extracellular transport of TeA toxin. 
growth rate did not change significantly. The above results showed that under nutrient-rich conditions, the growth of the $\triangle A a M F S 1$ strain was restricted, but on nutrient-deficient media, this restriction was released. In addition, $\triangle A a M F S 1$ also showed stronger UV resistance. Its spores still maintained a high germination rate after UV irradiation.

Mycotoxins are one of the main contributions to the virulence of A. alternata. $\triangle A a T A S 1$ lost the ability to produce TeA, so the infecting plaque on tomatoes was also significantly smaller. Unlike $\triangle A a T A S 1, \triangle A a M F S 1$ could produce a small amount of TeA, so its pathogenicity was not significantly affected. These findings indicate that low concentrations of TeA can help $A$. alternata complete the infection and colonization process in plants. In addition, $\triangle A a T A S 1$ and $\triangle A a M F S 1$ showed no significant changes in antioxidant capacity or cell-wall integrity compared with the wild type (Supplementary Fig. S3).

The differentially expressed genes revealed in the transcriptome explained the phenotypic differences. The synthetic precursors of TeA are L-Ile and acetoacetyl-CoA. In $\triangle A a T A S 1$, the TeA synthesis pathway was blocked. As a result, two synthetic precursors turned to other pathways. L-Ile affects amino acid metabolism (Bianchi et al. 2019). Acetoacetyl-CoA is synthesized by two molecules of acetyl-CoA catalyzed by thiolase (Fukao et al. 2019). Acetyl-CoA is an important precursor for the formation of fatty acids, ketone bodies, and other energy substances. It is also the product of fatty acid $\beta$-oxidation and pyruvate oxidative decarboxylation (Carrer et al. 2019). Therefore, knockout of AaTASI will have a certain impact on these aspects.

The transcriptome data of $\triangle A a M F S 1$ showed that the loss of the AaMFS1 gene affected the metabolism of basic elements, such as sulfur and nitrogen, and the metabolism of basic substances, such as purines, pyrimidines, pyruvate, and amino acids. This phenomenon might be related to the slow growth of $\triangle A a M F S 1$ on PDA plates. On MM, all groups were in a state of nutrient shortage, so the growth difference between $\triangle A a M F S 1$ and the other groups was reduced. In addition, we also used qPCR to confirm the expression levels of UV-induced damage response and repair-related genes. Some of them were significantly upregulated, corresponding to the phenotype of increased UV-resistance ability.

According to the expected functions of AaTAS1 and AaMFS1, we speculate that the AaTAS1 protein is responsible for the biosynthesis of TeA, so it should be mainly distributed in the cytoplasm. Moreover, the AaMFS1 protein is responsible for the transmembrane transport of TeA, so it should be distributed on the cell plasma membrane and the intracellular membrane system (Yun et al. 2020). The fluorescence localization results were consistent with the above hypothesis. Regarding the interaction between the two proteins, we speculate that the two proteins interact indirectly through TeA, which is supported by evidence showing that TeA suppressed the upregulation of AaMFS1 expression levels in $\triangle A a T A S 1$.

\section{MATERIALS AND METHODS}

\section{Source and culturing of $A$. alternata.}

The wild-type XJaa strain used in the experiment was obtained from jujube (Xinjiang, China). If not specified, XJaa were cultured on PDA plates at $25^{\circ} \mathrm{C}$ or in PDB liquid medium at $25^{\circ} \mathrm{C}$ and $150 \mathrm{rpm}$; E. coli were cultured on Luria-Bertani broth (LB) agar plates at $37^{\circ} \mathrm{C}$ or in $\mathrm{LB}$ broth liquid medium at $37^{\circ} \mathrm{C}$ and $220 \mathrm{rpm}$. The MM is used for starvation culture of A. alternata (Fu et al. 2020).

\section{Detection of mycotoxin TeA.}

Ultraviolet high-pressure liquid chromatography (UV-HPLC) (Thermo Fisher, Waltham, MA, U.S.A.) was used for the detection of mycotoxin TeA, and a $\mathrm{C} 18$ reversed-phase column $(4.6 \times 250 \mathrm{~mm}, 5 \mu \mathrm{m})$ (Thermo Fisher) was used to separate the substances. The mobile phase A was $0.1 \mathrm{M} \mathrm{NaH}_{2} \mathrm{PO}_{4}(\mathrm{pH} 3.2$, $\mathrm{H}_{3} \mathrm{PO}_{4}$ to adjust $\mathrm{pH}$ ), mobile phase $\mathrm{B}$ phase was methanol, the flow in was provided at the ratio of phase $\mathrm{A}$ to phase $\mathrm{B}=$ $50: 50$, the flow rate was $0.7 \mathrm{ml} / \mathrm{min}$, the UV detection wavelength was $277 \mathrm{~nm}$, and the peak of TeA was detected at approximately $8.8 \mathrm{~min}$. During this process, the column pressure was stabilized at approximately $120 \mathrm{bar}$, and the injection volume was generally $10 \mu \mathrm{l}$. We determined the same sample for six times, the relative standard deviation was less than $10 \%$, so the method has good repeatability (Supplementary Table S8). Furthermore, limit of detection (LOD) of this method is $0.25 \mathrm{ppm}$, and limit of quantitation (LOQ) is $0.5 \mathrm{ppm}$. In addition, we also used triple quadrupole liquid mass spectrometry to verify.

RT-qPCR confirms the relative expression level of genes.

RNAiso Plus (TaKaRa, Tokyo) was used to extract RNA from the hyphae. We used the PrimeScript RT reagent kit with gDNA Eraser (Takara) to reverse transcribe RNA. NovoStart SYBR qPCR SuperMix (Novoprotein, Shanghai, China) was used to configure the qPCR system. We used a QuantStudio 6 fluorescent qPCR (Applied Biosystems, Carlsbad, CA, U.S.A.) instrument for the measurements. After optimization of amplification efficiency, compatibility, and melting curves (Supplementary Table S9; Supplementary Fig. S10), the relative quantitative calculation method $\Delta \Delta \mathrm{Ct}$ is applied for analysis. The expression level change of the experimental group samples relative to the control group could be obtained.

\section{Transcriptome analysis.}

The experimental material used for the transcriptome consisted of mycelium cultured for 3 days in PDB. We extracted total RNA from experimental materials. The mRNA was separated from the total RNA using magnetic beads with Oligo dT, and small fragments of $300 \mathrm{bp}$ were separated after random fragmentation. Random primers were used for RT, and, after connecting the adaptors, they were sequenced on the Illumina NovaSeq 6000 platform. The clean data were screened using SeqPrep software. There were three biological replicates for each group of sequencing work. The clean reads were compared with the Altal 1 version of A. alternata_SRC1lrK2 $\mathrm{f}$ by Hisat2, and the number of reads was counted by RSEM. The significant difference analysis was conducted by DESeq2. Genes with an adjusted $P$ value less than 0.05 and an absolute value of a $\log _{2}$ fold change greater than 1 were classified as differentially expressed genes. $\mathrm{R}$ script was used to perform KEGG pathway enrichment analysis of the genes. When the adjusted $P$ value was less than 0.05 , the pathway was considered to be significantly enriched. The data were analyzed on the free online Majorbio Cloud Platform.

\section{A. alternata protoplast preparation.}

A small amount of $A$. alternata hyphae was smashed with a homogenizer. The breaking time was extended or the frequency increased as needed to eliminate obvious large mycelium clusters, followed by filtering with a single layer of sterile lenscleaning paper into a 50-ml centrifuge tube. Then, the sample was mixed with $25 \mathrm{ml}$ of PDB and was shaken overnight at $28^{\circ} \mathrm{C}$ and $220 \mathrm{rpm}$ until many tender mycelium clusters grew in the medium. After centrifugation at $3,220 \times g$ for $10 \mathrm{~min}$ to collect the mycelial precipitate, it was washed with $30 \mathrm{ml}$ of sterile water and $30 \mathrm{ml}$ of $0.7 \mathrm{M} \mathrm{NaCl}$ water, respectively. Then, $10 \mathrm{ml}$ of filtered and sterilized enzymatic solution (Driselase, lysozyme, and snailase, each $1 \%, 0.7 \mathrm{M} \mathrm{NaCl}$ dissolved) was added to the mycelial precipitate, and the mixture was incubated at $30^{\circ} \mathrm{C}, 100 \mathrm{rpm}$ for $5 \mathrm{~h}$. A few 
microliters of hydrolyzed liquid was collected for observation under a microscope to determine whether spherical protoplasts had formed. Sufficient double distilled $\mathrm{H}_{2} \mathrm{O}$ was added to observe whether the protoplasts would burst. After enzymolysis, the liquid was filtered with three layers of sterile lens-cleaning paper and was washed with $0.7 \mathrm{M} \mathrm{NaCl}$, and the filtrate was centrifuged at $3,220 \times g$ for $10 \mathrm{~min}$ at $4^{\circ} \mathrm{C}$. The piece of black precipitate at the bottom of the centrifuge tube was the desired protoplast. The precipitate was washed with STC (182.18 $\mathrm{g}$ of sorbital, $50 \mathrm{ml}$ of $1 \mathrm{M}$ Tris-HCl, $\mathrm{pH}$ 8.0, $7.351 \mathrm{~g}$ of $\mathrm{CaCl}_{2} \cdot 2 \mathrm{H}_{2} \mathrm{O}$ per liter), and, then, the concentration of the protoplasts was adjusted to $10^{7}$ per milliliter. The protoplasts were placed on ice for later use.

\section{A. alternata protoplast transformation.}

To $200 \mu \mathrm{l}$ of fresh protoplast fluid prepared on the same day, $2 \mu \mathrm{l}$ of a $5-\mathrm{mg} / \mathrm{ml}$ heparin sodium solution, $5 \mu \mathrm{g}$ of the knock-out fragment for gene knockout, or $2 \mu \mathrm{l}$ of a $5-\mathrm{mg} / \mathrm{ml}$ heparin sodium solution, $15 \mu \mathrm{g}$ of recombinant plasmid, and $5 \mu \mathrm{l}$ of salmon sperm DNA at $2 \mathrm{mg} / \mathrm{ml}$ was added for gene complementation, followed by gentle mixing, allowing the mixture to stand on ice for 30 min, slowly adding $1 \mathrm{ml}$ of SPTC (40\% PEG4000 in STC solution) dropwise with gentle mixing and allowing the mixture to stand at room temperature for $20 \mathrm{~min}$. The entire transformation mixture was added to $10 \mathrm{ml}$ of TB3 solution $(3 \mathrm{~g}$ of yeast extract, $3 \mathrm{~g}$ of casein acid hydrolysate, $200 \mathrm{~g}$ of sucrose per liter, $50 \mu \mathrm{g}$ ampicillin per milliliter) and was incubated overnight at $30^{\circ} \mathrm{C}, 70 \mathrm{rpm}$ on a shaker, after which some young mycelia were obtained. We added $10 \mathrm{ml}$ of $0.7 \mathrm{M} \mathrm{NaCl}$ to the liquid and, then, centrifuged the sample at $3,220 \times g$ for 10 min to obtain hyphal precipitates; $500 \mu \mathrm{l}$ of the liquid was left to spread on 10-ml TB3 $1.5 \%$ agar plates (ampicillin at $50 \mu \mathrm{g} / \mathrm{ml}$, hygromycin at a final concentration of $50 \mu \mathrm{g} / \mathrm{ml}$, or $\mathrm{G} 418$ at a final concentration of $50 \mu \mathrm{g} / \mathrm{ml}$ ). After incubating the plates at $25^{\circ} \mathrm{C}$ for $10 \mathrm{~h}$, we poured 45 to $50^{\circ} \mathrm{C}$ TB3 $2 \%$ agar (ampicillin at $50 \mu \mathrm{g} / \mathrm{ml}$, hygromycin at a final concentration of $100 \mu \mathrm{g} / \mathrm{ml}$, or $\mathrm{G} 418$ at a final concentration of $100 \mu \mathrm{g} / \mathrm{ml}$ ) to seal the plate, inverted the plate, and incubated it at $25^{\circ} \mathrm{C}$ for 3 to 5 days to obtain transformants. The identification primers for the target genes or the resistance gene could be used for genotype identification.

\section{Heterologous expression \\ of A. alternata AaTAS1-A domain protein.}

Primers were designed to amplify the AaTAS1-A domaincoding DNA sequence from the total complementary DNA of A. alternata, and a one-step cloning kit (Vazyme Biotech, Nanjing, China) was used to ligate pET28a under the principle of homologous recombination. Then, following complete plasmid amplification by $\mathrm{DH} 5 \alpha$, the purified plasmid was transformed into E. coli BL21(DE3) cells, and the bacterial solution was cultivated until the optical density at $600 \mathrm{~nm}$ was 0.5 . To induce protein expression, $0.05 \mathrm{mM}$ isopropyl- $\beta$-D-thiogalactoside was added to the medium, at $18^{\circ} \mathrm{C}, 180 \mathrm{rpm}$ overnight. After centrifugation to obtain the bacterial precipitate, it was washed and suspended in phosphate buffered saline solution, after which ultrasonication was used to disrupt the bacterial cells. After centrifugation at $13,800 \times g$ and $4^{\circ} \mathrm{C}$ for $30 \mathrm{~min}$, the supernatant was collected and purified by affinity chromatography on a nickel column (Sangon, Shanghai, China). The broken bacterial liquid, supernatant, loading elution, washing elution, and eluate passed through a nickel column were collected as samples. The protein samples were added to $6 \times$ sodium dodecyl sulfate-polyacrylamide gel electrophoresis (SDS-PAGE) loading buffer (Beyotime, Shanghai, China), were boiled at $100^{\circ} \mathrm{C}$ for $5 \mathrm{~min}$, and were then loaded in a 10- $\mu$ l volume for SDS-PAGE. The gel was stained with Coomassie brilliant blue (Beyotime), the eluate containing the target protein was collected, and an ultrafiltration membrane with a $10-\mathrm{kDa}$ pore size (Beyotime) was used to concentrate the protein.

\section{AaTAS1-A domain protein function assay.}

The working buffer composition consisted of $50 \mathrm{mM}$ Tris$\mathrm{HCl}, \mathrm{pH} 7.5,10 \mathrm{mM} \mathrm{MgCl}_{2}$, and $1 \mathrm{mM}$ dithiothreitol. We made protein concentrations in the reaction system to be 0,50 , and $100 \mu \mathrm{g} / \mathrm{ml}$, adding ATP and L-Ile to obtain final concentrations of $0.5 \mathrm{mM}$. Finally, the reaction system was incubated at $25^{\circ} \mathrm{C}$ for $15 \mathrm{~min}$. A PPi detection kit (AAT Bioquest, Sunnyvale, CA, U.S.A.) was used for detection.

\section{Statistics.}

At least three biological replicates of each group were performed in all above assays. Values in the column chart are the standard deviation. The Student's $t$ test and one-way analysis of variance were used to test whether there were significant differences among the different groups.

\section{Accession numbers.}

All relevant data are within the manuscript and the Supplementary Files. The RNA-seq raw data have been deposited in the National Center for Biotechnology Information (NCBI) database (BioProject ID: PRJNA781145 [Run IDs: SRR16964232 to SRR16964237] and PRJNA781278 [Run IDs: SRR16970016 to SRR16970021]). De novo sequencing raw data have been deposited in the NCBI database (BioProject ID: PRJNA784742 [Run IDs: SAMN23500129 to SAMN23500134]).

\section{ACKNOWLEDGMENTS}

We thank the Institutional Center for Shared Technologies and Facilities of SINH, CAS for technical assistance. We are also deeply grateful to C. Jiang (Northwest A\&F University, China) and Y. Chen (Zhejiang University, China) for helpful discussions.

\section{LITERATURE CITED}

Aichinger, G., Del Favero, G., Warth, B., and Marko, D. 2021. Alternaria toxins-Still emerging? Compr. Rev. Food Sci. Food Saf. 20:43904406.

Bianchi, F., Van't Klooster, J. S., Ruiz, S. J., and Poolman, B. 2019. Regulation of amino acid transport in Saccharomyces cerevisiae. Microbiol. Mol. Biol. Rev. 83:e00024-19.

Bills, G. F., and Gloer, J. B. 2016. Biologically active secondary metabolites from the fungi. Microbiol. Spectr. 4. Published online.

Carrer, A., Trefely, S., Zhao, S., Campbell, S. L., Norgard, R. J., Schultz, K. C., Sidoli, S., Parris, J. L. D., Affronti, H. C., Sivanand, S., Egolf, S., Sela, Y., Trizzino, M., Gardini, A., Garcia, B. A., Snyder, N. W., Stanger, B. Z., and Wellen, K. E. 2019. Acetyl-CoA metabolism supports multistep pancreatic tumorigenesis. Cancer Discov. 9:416-435.

Corre, C., and Challis, G. L. 2009. New natural product biosynthetic chemistry discovered by genome mining. Nat. Prod. Rep. 26:977-986.

Davis, N. D., Diener, U. L., and Morgan-Jones, G. 1977. Tenuazonic acid production by Alternaria alternata and Alternaria tenuissima isolated from cotton. Appl. Environ. Microbiol. 34:155-157.

Fraeyman, S., Croubels, S., Devreese, M., and Antonissen, G. 2017. Emerging Fusarium and Alternaria mycotoxins: Occurrence, toxicity and toxicokinetics. Toxins 9:228.

Fu, H., Chung, K. R., Gai, Y., Mao, L., and Li, H. 2020. The basal transcription factor II $\mathrm{H}$ subunit $\mathrm{Tfb} 5$ is required for stress response and pathogenicity in the tangerine pathotype of Alternaria alternata. Mol. Plant Pathol. 21:1337-1352.

Fukao, T., Sasai, H., Aoyama, Y., Otsuka, H., Ago, Y., Matsumoto, H., and Abdelkreem, E. 2019. Recent advances in understanding betaketothiolase (mitochondrial acetoacetyl-CoA thiolase, T2) deficiency. J. Hum. Genet. 64:99-111.

Kim, L. J., Ohashi, M., Zhang, Z., Tan, D., Asay, M., Cascio, D., Rodriguez, J. A., Tang, Y., and Nelson, H. M. 2021. Prospecting for natural products by genome mining and microcrystal electron diffraction. Nat. Chem. Biol. 17:872-877. 
Kumar, N., Raja, S., and Van Houten, B. 2020. The involvement of nucleotide excision repair proteins in the removal of oxidative DNA damage. Nucleic Acids Res. 48:11227-11243.

Lee, H. B., Patriarca, A., and Magan, N. 2015. Alternaria in food: Ecophysiology, mycotoxin production and toxicology. Mycobiology 43:93106.

Motoyama, T., Yun, C. S., and Osada, H. 2021. Biosynthesis and biological function of secondary metabolites of the rice blast fungus Pyricularia oryzae. J. Ind. Microbiol. Biotechnol. 48:kuab058.

Oliveira, R. C., Goncalves, S. S., Silva, C. D. C., Dilkin, P., Madrid, H., and Correa, B. 2019. Polyphasic characterization of Epicoccum sorghinum: A tenuazonic acid producer isolated from sorghum grain. Int. J. Food Microbiol. 292:1-7.

Puntscher, H., Hankele, S., Tillmann, K., Attakpah, E., Braun, D., Kütt, M. L., Del Favero, G., Aichinger, G., Pahlke, G., Höger, H., Marko, D., and Warth, B. 2019. First insights into Alternaria multi-toxin in vivo metabolism. Toxicol. Lett. 301:168-178.

Quistgaard, E. M., Löw, C., Guettou, F., and Nordlund, P. 2016. Understanding transport by the major facilitator superfamily (MFS): Structures pave the way. Nat. Rev. Mol. Cell Biol. 17:123-132.

Rowe, L. A., Degtyareva, N., and Doetsch, P. W. 2012. Yap1: A DNA damage responder in Saccharomyces cerevisiae. Mech. Ageing Dev. 133:147156.

Scherlach, K., and Hertweck, C. 2021. Mining and unearthing hidden biosynthetic potential. Nat. Commun. 12:3864.

Schwarz, C., Kreutzer, M., and Marko, D. 2012. Minor contribution of alternariol, alternariol monomethyl ether and tenuazonic acid to the genotoxic properties of extracts from Alternaria alternata infested rice. Toxicol. Lett. 214:46-52.
Scopes, R. K. 2001. Overview of protein purification and characterization. Pages B4.1.1-B4.1.9 in: Current Protocols in Protein Science. John Wiley \& Sons, New York.

Sinha, R. P., and Häder, D. P. 2002. UV-induced DNA damage and repair: A review. Photochem. Photobiol. 1:225-236.

Stanišić, A., and Kries, H. 2019. Adenylation domains in nonribosomal peptide engineering. Chembiochem 20:1347-1356.

Stickings, C. E., and Townsend, R. J. 1961. Studies in the biochemistry of micro-organisms. 108. Metabolites of Alternaria tenuis Auct.: The biosynthesis of tenuazonic acid. Biochem. J. 78:412-418.

Stinson, E. E. 1985. Mycotoxins-Their biosynthesis in Alternaria. J. Food Prot. 48:80-91.

Yan, N. 2015. Structural Biology of the Major Facilitator Superfamily Transporters. Annu. Rev. Biophys. 44:257-283.

Yun, C. S., Motoyama, T., and Osada, H. 2015. Biosynthesis of the mycotoxin tenuazonic acid by a fungal NRPS-PKS hybrid enzyme. Nat. Commun. 6:8758

Yun, C. S., Motoyama, T., and Osada, H. 2017. Regulatory mechanism of mycotoxin tenuazonic acid production in Pyricularia oryzae. ACS Chem. Biol. 12:2270-2274.

Yun, Y., Guo, P., Zhang, J., You, H., Guo, P., Deng, H., Hao, Y., Zhang, L., Wang, X., Abubakar, Y. S., Zhou, J., Lu, G., Wang, Z., and Zheng, W. 2020. Flippases play specific but distinct roles in the development, pathogenicity, and secondary metabolism of Fusarium graminearum. Mol. Plant Pathol. 21:1307-1321.

Zhou, J., Chi, X., Cheng, M., Huang, X., Liu, X., Fan, J., Xu, H., Lin, T., Shi, L., Qin, C., and Yang, W. 2019. Zika virus degrades the $\omega-3$ fatty acid transporter Mfsd2a in brain microvascular endothelial cells and impairs lipid homeostasis. Sci. Adv. 5:eaax7142. 